Théologiques

Théologiques

\title{
La solidarité et la justice reconsidérées
}

\section{Hille Haker}

Volume 22, numéro 1, 2014

Vivre dans la diversité

URI : https://id.erudit.org/iderudit/1033091ar

DOI : https://doi.org/10.7202/1033091ar

Aller au sommaire du numéro

\section{Éditeur(s)}

Faculté de théologie et de sciences des religions, Université de Montréal

\section{ISSN}

1188-7109 (imprimé)

1492-1413 (numérique)

Découvrir la revue

\section{Citer cet article}

Haker, H. (2014). La solidarité et la justice reconsidérées. Théologiques, 22(1), 13-25. https://doi.org/10.7202/1033091ar

\section{Résumé de l'article}

En commençant par déplorer l'absence de place pour la solidarité dans l'interprétation de la "justice de marché " au XxI ${ }^{\mathrm{e}}$ siècle, l'article propose une autre voie, soit la relation dialectique entre la justice et la solidarité. Alors que la justice met l'accent sur l'« égalité » pour tous, la solidarité s'attarde surtout sur la " différence » et la diversité. Le pont entre les deux concepts peut être trouvé dans les récentes réinterprétations de la théorie de la reconnaissance, qui touche les sphères privée, sociale et politique. L'article préconise toutefois une approche critique, qui prend le manque de reconnaissance, plutôt que la reconnaissance, comme point de départ. L'éthique en général et l'éthique chrétienne en particulier doivent prêter attention aux expériences et aux récits des personnes qui semblent invisibles et inaudibles dans les systèmes de justice fondés sur le mérite et les accomplissements, et ce, dans le but d'identifier les violations de droits aussi bien que les pratiques d'exclusion subies par ces personnes. En se préoccupant d'abord des expériences réelles d'injustice au lieu de la théorie normative de la justice, la relation dialectique entre la justice et la solidarité devient claire : la solidarité révèle non seulement le fossé entre la théorie et la pratique, ou l'inévitable côté aveugle de la justice, mais elle s'affiche aussi clairement en faveur des personnes souffrant d'injustice. La solidarité est orientée vers l'action. Elle fait pression sur les institutions pour qu'elles deviennent justes, tout en prônant des changements pour ceux et celles qui souffrent le plus d'injustice, et tout en accompagnant ces personnes. Par ailleurs, la solidarité critique mais aussi partisane nécessite la vision impartiale et égalitaire d'une justice en tant que critique d'une idéologie potentielle qui est liée à un concept identitaire et à une politique identitaire. 


\title{
La solidarité et la justice reconsidérées*
}

\author{
Hille HAKeR * * \\ Chaire d'éthique Richard McCormick S.J. \\ Université Loyola (Chicago, USA)
}

«Seulement cinq euros, rien que cinq euros ", dit à voix basse Maria, une jeune prostituée aux joues creuses et aux cheveux débraillés, qui vient de surgir d'un sombre passage couvert de graffitis au centre d'Athènes lors d'une soirée récente.

Au moment où un vent frais fait s'envoler des morceaux de papier et des déchets au-dessus d'un trottoir crasseux, Angelos Tzortzinis, un photographe grec, aperçoit Maria en train d'abaisser son prix pour l'équivalent de 6,50\$. Maria, un pseudonyme qu'elle se donne, avait espéré obtenir de l'argent pour manger - dans une nouvelle rue où la prostitution est bon marché mais dangereuse en raison du trafic de drogue, un endroit que l'on a commencé à fréquenter avec la crise en Grèce, et qui garantit à Maria de pouvoir oublier ses soucis, ne serait-ce que pour un moment.

Le pays entrant dans sa cinquième année de dépression économique, et le taux chômage chez les jeunes frôlant les 60 \%, de plus en plus de femmes et d'hommes offrent leur corps pour une somme infime et dérisoire. Selon le Centre national de recherche en sciences sociales, le nombre de travailleurs et travailleuses du sexe a grimpé de $150 \%$ au cours des deux dernières années.

De nombreuses personnes se livrant à la prostitution vendent leurs services pour un faible montant, qui varie de 10 à 15 euros, un prix qui a fondu au

Article traduit de l'anglais par Richard Côté.

** Hille Haker est titulaire de la Richard McCormick S.J. Chair of Ethics à l'Université Loyola de Chicago, depuis 2010. Elle est membre du Groupe européen d'éthique des sciences et des nouvelles technologies (GEE) depuis 2005, membre du conseil d'administration de plusieurs revues et membre du comité de rédaction de Concilium. Elle a récemment publié (2011) Hauptsache gesund, Kösel Verlag, et codirigé (2013) Religiöser Pluralismus in der Klinikseelsorge, Berlin/Münster, LIT Verlag. Elle travaille actuellement sur un ouvrage à paraître: Recognition and Responsibility: Critical Theory and Christian Ethics.

C Revue Théologiques 2014. Tout droit réservé. 
même rythme que le revenu des clients, touché par la crise. Beaucoup plus qu'avant, ces personnes mettent leur santé en danger en ayant des relations sexuelles non protégées, qu'elles offrent pour une somme supplémentaire. En outre, la violence et les viols sont à la hausse.

Enfin, une nouvelle menace est apparue: une sorte de méthamphétamine cristalline appelée "chicha ", d'après le narguilé turc, mais connue aussi comme la "cocaïne du pauvre », obtenue par un mélange de barbituriques et d'ingrédients incluant l'alcool, le chlore et même l'acide contenu dans les batteries.

$[\ldots]$

Généralement, la chicha se fume. Mais, on la prend de plus en plus par voie intraveineuse. À cause des produits chimiques toxiques qu'elle renferme, un nombre croissant d'usagers aboutissent à l'urgence. Les experts en santé publique jugent aussi que les injections ont contribué à la hausse alarmante des cas de VIH partout en Grèce, qui ont grimpé de 50 \% depuis 2011, alors que le nombre de personnes consommant des stupéfiants a augmenté.» (New York Times, 21 mai 2013, traduit de l'anglais)

L'histoire de Maria illustre bien les derniers développements au sein de l'Union européenne, c'est-à-dire les hauts taux de pauvreté et de chômage dans des pays que la crise financière a frappé durement, et qui doivent maintenant faire face à des politiques d'austérité que l'on dit nécessaires, non seulement au nom d'une prospérité à recouvrer mais aussi au nom de la justice. Les pays où ces politiques sont appliquées - Grèce, Espagne, Portugal, Italie, Irlande et Chypre - ne peuvent poursuivre des activités économiques nationales d'après le modèle du capitalisme financier à moins de démontrer leur volonté d'apporter des réformes socio-économiques qui s'attaquent à leur dette nationale. Ces réformes, que la majorité des États de l'Union européenne appliquent - y compris les pays mentionnés précédemment —, seraient nécessaires non seulement pour sauver la zone euro, mais seraient également justes. Au moins pour le temps présent, on ne pourrait faire autrement que d'avoir de telles mesures, qui affectent la justice sociale, avec pour résultat des taux de chômage élevés et l'augmentation de la pauvreté, de la maladie, du nombre de sans-abri, et aussi de l'écart entre les nantis et les moins nantis. On a espoir bien sûr qu'après un temps de récession, les économies nationales se rétabliront, donnant une nouvelle chance aux citoyens européens, incluant ceux habitant la partie sud de l'Europe, de prospérer. 
Maria cependant ne connaîtra peut-être pas ce futur, si jamais il advient. Et même si elle y parvient, les blessures qu'elle a subies pourrontelles guérir? Ou encore, on peut s'attendre à ce qu'elle aura perdu en grande partie son estime de soi, sa reconnaissance sociale, sa santé et son aptitude à intégrer un marché du travail peu propice aux plus pauvres d'entre les pauvres - mais alors, demandera-t-on, qu'arrivera-t-il aux itinérants aux prises avec un problème de drogue, qui auront peut-être contracté le VIH/SIDA, et qui, comme Maria, n'auront réussi à survivre qu'en vendant leur corps? Que signifie la justice pour Maria et les millions de femmes, d'hommes et d'enfants qui constituent ce qu'on appelle le «milliard inférieur» - les plus pauvres d'entre les pauvres, les personnes les moins reconnues, les moins respectées sur Terre? «To speak of God means to express the suffering of others and to lament responsibility neglected and solidarity refused" (Metz 1999, 231).

Quelle serait la conséquence pour la théologie chrétienne de comprendre le concept de solidarité comme Metz l'entend? Car pour lui la théo-logie n'a pas de sens si elle ne se préoccupe pas de la souffrance, ni ne se plaint du manque de responsabilité et du refus de solidarité. Dans les remarques qui suivent, je signalerai quelques éléments d'un modèle d'éthique politique chrétienne qui serait dans l'esprit de la théologie politique de Metz - avec l'intention de situer notre réflexion concernant la diversité dans un contexte de justice et de solidarité, au lieu de simplement discuter de ce sujet en lien avec l'attitude libérale de tolérance envers les étrangers dans les sociétés pluralistes.

Une éthique chrétienne et une théologie qui suivraient la nouvelle théologie politique de Metz débuteraient par la prise en compte des souffrances vécues, qui appellent à la responsabilité et à la solidarité, mais dont on ne tient pas compte. C'est une éthique qui critique les pratiques et politiques qui n'accordent aucune place à la responsabilité et à la solidarité. C'est une éthique qui replace dans leur contexte les revendications d'ordre éthique par le recours aux récits des personnes qui, comme Maria dans l'article du New York Times, sont non reconnues, réduites au silence et ignorées par les politiques visant à rétablir le statu quo d'une économie de marché injuste. C'est aussi une éthique qui cherche à formuler des propositions constructives découlant de sa propre tradition et qui prône une société se définissant comme une communauté diversifiée, préoccupée par la justice et la solidarité.

Le concept de justice implicite qui dicte les politiques de l'Union européenne, comme celles de la plupart des politiques touchant à la pauvreté, 
est essentiellement défini en termes économiques: il est plutôt fondé sur le "mérite» qui distingue entre eux les individus et les groupes, au lieu d'être fondé sur les besoins (fondamentaux) qui sont les mêmes pour tous les individus; ils apparaissent dans la Déclaration des droits de la personne, et ils vont des besoins élémentaires (alimentation, logement, travail) aux libertés fondamentales, et jusqu'à la possibilité de participer à la conduite des affaires sociales et politiques. Cependant, les politiques actuelles sont plus orientées vers la stabilité des marchés que vers la stabilité sociale, ce qui résulte en la prédominance de la «justice de marché» sur la «justice sociale». La justice de marché semble structurellement indifférente aux conséquences sociales des réformes financières; son interprétation implicite de la justice - où les occasions sont offertes aux personnes qui le méritent - est pratiquement étrangère au concept de solidarité. C'est un concept qui n'a pas grand-chose en commun avec l'attitude judéo-chrétienne devant la souffrance ni avec la promesse du Royaume de Dieu - à savoir une justice qui vient: premièrement de l'alliance entre Dieu et toutes les personnes; deuxièmement de la reconnaissance inconditionnelle de chaque personne, même si elle est non reconnue au sein de sa culture ou de sa société; et troisièmement de la compassion qui nécessite de transformer la souffrance en bien-être, de convertir l'injustice en justice, et l'exclusion en inclusion. Ce concept de justice ne repose pas sur un contrat économique, qui proclame que les règles sont les mêmes pour tous, mais qui dissimule que, dans le monde socio-économique actuel, les inégalités structurelles sont dans les faits tolérables. Ce concept de justice repose plutôt sur un contrat social qui confirme que toutes les personnes sont égales, indépendamment des différences qui existent entre elles, tout en ayant conscience que quelques-unes de ces différences proviennent d'inégalités intolérables au sein de sociétés qui visent à établir la justice.

On pourrait trouver biaisé mon bref aperçu des politiques européennes ou internationales, qui donne un caractère économique à la justice - un caractère qui favorise la distribution des biens matériels et des biens sociaux immatériels selon l'effort et la réussite, plutôt que selon les besoins et les droits. Mais toute théorie sur la justice ne peut être que préoccupée par le clivage entre le principe normatif affirmant l'égalité entre toutes les personnes et les diverses inégalités structurelles observées dans la réalité, qui touchent à l'appartenance sociale, culturelle, ethnique ou religieuse des individus. La tâche de toute théorie sur la justice consiste alors à expliquer comment l'affirmation normative au sujet de l'égalité peut être traduite en politiques qui combattent vraiment les inégalités, sans ignorer la reconnais- 
sance du droit à la différence pour autrui. L'égalité n'empêche pas la diversité - mais le problème consiste à éviter que la différence serve à masquer et à tolérer l'inégalité, c'est-à-dire l'injustice. C'est sur ce point que la relation entre la justice et la solidarité prend tout son sens.

John Rawls, un des plus importants théoriciens de la justice du $\mathrm{xx}^{\mathrm{e}}$ siècle, a proposé un principe de la différence, formulant que les «social and economic inequalities are to be arranged so that they are to be of the greatest benefit of the least-advantaged members of society ". Il s'agissait d'un complément au principe d'égalité, qui énonce que «each person is to have an equal right to the most extensive basic liberty compatible with a similar liberty for others» (Rawls 1999) ${ }^{1}$. Mais plusieurs commentateurs ne l'ont pas suivi sur cette question. Rawls aurait pu appeler son principe de différence le principe de solidarité lié à la justice car, pour les citoyens d'une société donnée, il sert de compensation aux injustices structurelles ou aux situations de départ inégales, qui entraînent des possibilités inégales de prospérer ou d'avoir "des rêves de vie meilleure ", ou les deux. Cependant, alors que le concept libéral d' «accès égalitaire » aux richesses de la société, tel que développé dans le premier principe de justice, a été bien accueilli par les penseurs occidentaux en philosophie politique, la dimension de la justice touchant à la solidarité a été systématiquement négligée, ou carrément rejetée.

Comme résultat, nous nous retrouvons aujourd'hui à accepter que notre économie mondialisée ne réserve de place ni à un principe ni à l'autre: la norme fondamentale d'une société juste, qui donne à chaque personne le même droit aux libertés fondamentales, apparaît comme une utopie n'ayant pas sa place au $\mathrm{XxI}^{\mathrm{e}}$ siècle. Et on avance que, même si c'est possible en théorie, on ne peut traduire cette norme en véritables politiques dans un contexte de mondialisation. En outre, la revendication voulant que l'inégalité soit acceptable seulement quand elle profite aux personnes les moins avantagées a été remplacée par une compréhension élargie de la

1. D'autres modifications apparaissent dans Political Liberalism, où on peut lire ceci: "Each person has an equal claim to a fully adequate scheme of equal basic rights and liberties, which scheme is compatible with the same scheme for all; and in this scheme the equal basic liberties, and only those liberties, are to be guaranteed their fair value." Le principe modifié devient alors: "Social and economic inequalities are to satisfy two conditions : first, they are to be attached to positions and offices open to all under conditions of fair equality of opportunity; and second, they are to be to the greatest benefit of the least advantaged members of society» (Rawls 2013, 5-6). 
différence et de la diversité qui ignore précisément la distinction entre une différence tolérée et une injustice tolérée.

Mais où se situent la théologie et l'éthique chrétiennes dans ce débat? En théorie, nous avons besoin de préciser comment la justice est liée à la solidarité. En pratique, cependant, cela entraîne que la communauté de foi chrétienne a besoin de montrer que la justice comme la solidarité peuvent être accomplies. Ma thèse est la suivante: les deux sont possibles seulement quand on ajoute un troisième terme servant à expliquer pourquoi il ne faut pas séparer la justice de la solidarité. Ce terme est «reconnaissance » et il est présent de nos jours dans la théorie politique et dans l'éthique. L'éthique chrétienne peut également l'adopter, et ce, à partir de sa propre tradition, qui veut que Dieu accorde inconditionnellement la même importance à chaque personne, peu importe son rang social ou économique. Ce qui est nécessaire pour l'interprétation de la justice et de la solidarité, et de leur interrelation, c'est la reconnaissance de chaque individu comme étant une personne - et seulement une - parmi d'autres. Selon un concept développé par Axel Honneth, par exemple, la tâche de l'éthique consiste à analyser les structures de l'injustice en fonction de la non-reconnaissance, qui se traduit par le manque de respect, la discrimination sociale et le déni des droits politiques. Par conséquent, il juge que le travail de l'éthique consiste à lutter contre le manque de reconnaissance dans les relations interpersonnelles, les structures sociales et les institutions politiques. Il souhaite de plus que l'on inclue le souci pour autrui et la solidarité comme étant l'«autre côté » de la justice².

Il m'apparaît assurément possible de défendre l'analyse de Honneth, mais dans son concept emprunté à Hegel, la «lutte pour la reconnaissance» est encore laissée aux personnes qui souffrent d'un manque de reconnaissance - son succès ou son échec dépendant plus de la solidarité parmi les personnes souffrant d'injustice que sur une révision de l'interprétation des devoirs dictés par la morale. Cette analyse doit se comprendre comme un plaidoyer en faveur des personnes subissant une injustice, et comme une solidarité avec elles, conjointement avec une solidarité au sein même de ces personnes. Afin d'illustrer mon point de vue au sujet du rôle de la reconnaissance, dans le lien entre la justice et la solidarité, laissez-moi exposer une idée mise de l'avant par Paul Ricœur dans un de ses derniers

2. Honneth prend le concept hégélien comme point de départ pour une théorie de la liberté qui n'est pas seulement une variante d'une théorie politique libérale, mais plutôt une solution de rechange fondée sur la théorie de la reconnaissance. 
essais, en commençant par une citation qu'il tire d'un texte de Thomas Nagel. Nagel traite des points de vue personnels, que l'on ne peut remplacer par un "point de vue de nulle part» (a view from nowhere), comme il le soutient dans son célèbre livre où apparaît cette expression dans le titre. Cependant, Nagel avance que l'on peut en arriver à une vision commune en tenant compte de ce point de vue personnel:

Each of us begins with a set of concerns, desires, and interests of his own, and each of us can recognize that the same is true of others. We can remove ourselves in thought from our particular position in the world and think simply of all those people, without singling out as $I$ the one we happen to be. (Nagel 1991, 10, cité dans Ricœur 2007, 67)

Cette capacité de se distancier de ses propres désirs et points de vue est la prémisse pour pouvoir faire un jugement moral - Ricœur l'appelle la prémisse "anthropologique». Comme l'avance Ricœur, ce mouvement de va-et-vient entre mon point de vue personnel et un point de vue impersonnel, où je suis un parmi tous les autres, n'est pas différent de la capacité de prioriser mes divers désirs en fonction des «évaluations fortes " proposées par Charles Taylor, et qui sont pour lui un processus de balancier entre mes différents désirs, ou entre les valeurs que je cherche à développer - en définitive, tout cela est lié au concept d'identité morale idéale 3 . L'ordre des désirs ou des intérêts dans le concept de Taylor s'inspire de l'idée d'un moi idéalisé. Bien sûr, mes intérêts ne sont pas indépendants de ma position sociale, de ma culture, de ma religion ou du contexte historique où j'évolue - mais ce qui importe, c'est qu'ils soient en lien avec un idéal d'identité personnelle, qui coïncide avec l'identité morale dans la pensée de Taylor. Selon Ricœur, toutefois, cette identité morale coïncide non pas avec l'association entre des évaluations fortes et une identité personnelle idéalisée, mais avec cette identité qui inclut le point de vue de la justice. Par conséquent, si une «négociation" ou un jeu de balancier, ou quelque chose de semblable, se produit entre mes divers intérêts, quand j'oscille entre mon point de vue personnel et un point de vue impersonnel, c'est seulement alors que ce processus prend un caractère de négociation morale - ce qui est précisément la capacité morale de faire ce qu'on devrait faire - , c'està-dire de juger "without singling out as $I$ the one we happen to be ». C'est donc ce qui nous rend capables de lier notre désir de bien vivre avec le besoin d'être juste. Pour Ricœur, cette manière de porter un jugement est

3. Pour une analyse approfondie de la démonstration de Taylor, voir Haker (1999). 
en effet une négociation, entre moi-même et moi-même (pour ainsi dire) ou entre différents acteurs. Et ce processus de négociation implique assurément la présence de conflits entre divers désirs et intérêts, de conflits entre le point de vue personnel et le point de vue impartial, égalitaire, ainsi que de conflits entre différents acteurs. Sans surprise, ce sont pour Ricœur des conflits d'interprétation - à propos des faits comme à propos des normes.

Je soutiens la vision de Ricœur ainsi: si nous pouvions au moins nous mettre d'accord sur l'idée que nous avons cette capacité d'agir moralement en transcendant notre point de point de vue personnel, nous pourrions commencer le processus de négociation sur la meilleure voie à prendre, c'est-à-dire celle qui conduirait à combattre l'injustice dans une situation donnée.

Pourquoi la reconnaissance doit-elle jouer un rôle dans ce processus ? La première réponse donnée par Honneth, Taylor, Judith Butler, Martha Nussbaum et plusieurs autres est celle-ci: la reconnaissance est une condition importante pour le bien-être d'une personne. Comme Axel Honneth l'a montré, la reconnaissance interpersonnelle, sociale et légale est étroitement liée à l'identité individuelle, sociale et politique. Sans elle, l'intégrité morale et physique d'une personne pourrait être mise en péril et même se détériorer, son appartenance sociale étant affectée ou brisée, et ses droits politiques étant bafoués. On peut en observer les répercussions de multiples façons: violence physique ou psychologique, exclusion sociale et négation de certains droits. Une personne subissant une violence sur une longue période de temps court en effet le risque de voir son identité ravagée. Une personne - ou une communauté - étiquetée comme "différente ", car elle présente des caractéristiques particulières (pouvant toucher à la religion, à la culture, au sexe, à l'appartenance ethnique, etc.), et qui connaît une perte de reconnaissance sociale, cette personne ou cette communauté est de fait exclue des multiples activités dont dépend la cohésion d'une société4. En outre, pour reprendre les célèbres paroles de Hannah

4. Une analyse très impressionnante des effets du manque d'appartenance se retrouve, comme c'est souvent le cas, dans une œuvre de fiction, soit celle de Richard Power, The Time of Our Singing (trad. en français: Le temps où nous chantions). Ce roman relate le combat d'une famille américaine de race «hybride» du $\mathrm{xx}^{\mathrm{e}}$ siècle, où la société requiert une absence d'ambiguité pour déterminer l' «appartenance » sociale — bien que l' «appartenance» raciale elle-même crée de multiples injustices: les enfants dits métissés semblent aux prises avec une injustice de type racial en plus d'un manque de reconnaissance sociale venant de leur identité particulière. 
Arendt, sans «le droit d'avoir des droits", le statut de citoyen est impossible à garder et il ne peut être pratiqué - le droit d'avoir des droits devient alors un préalable à l'égalité dans la sphère politique.

Si ce dernier aspect de la justice, qui concerne les droits politiques de chaque personne, sans exception, a été au centre de la philosophie politique du $\mathrm{xx}^{\mathrm{e}}$ siècle, la théorie de la reconnaissance a quant à elle exposé le lien entre cette reconnaissance politique et les deux autres types, soit les reconnaissances sociale et interpersonnelle. On pourrait bien entendu rétorquer qu'il est difficile pour une éthique de la justice de porter son attention sur la violence contre la personne et sur l'exclusion sociale - et pourtant, il faut en tenir compte, par exemple quand l'État intervient dans un cas de violence conjugale, quand ces problèmes entrainent des frais médicaux, etc. En outre, l'exclusion sociale doit être combattue sur divers plans par des politiques antidiscriminatoires, et la cohésion sociale doit être favorisée par des mesures d'inclusion. Mais en définitive, aucune intervention judiciaire ne peut remplacer les besoins en fraternité, en inclusion et en plaidoyer nécessaires pour enrayer l'injustice à l'intérieur de la société civile et des institutions sociales. La solidarité est précisément cette pratique qui consiste à fraterniser avec les personnes jetées dans la honte, à inclure les personnes qu'on a socialement exclues, et elle est la voix politique et légale des personnes qui n'ont aucune voix. La justice a besoin de gestes de solidarité afin de discerner les pratiques où la diversité repose sur l'injustice. Exprimer une injustice subie — par l'entremise d'un récit — est une étape nécessaire dans le processus de négociation entre divers intérêts. Mais la solidarité a également besoin du point de vue de la justice afin d'aller au-delà du point de vue nécessairement partial des politiques identitaires sur lesquelles se fonde souvent la solidarité.

En prenant en considération les personnes que nos politiques contemporaines oublient et que nos sociétés civiles marginalisent, de même que les personnes qui n'apparaissent plus aux yeux des membres de leur communauté, des femmes comme Maria vont ré-apparaître dans l'espace public. Souvent, ces personnes n'ont pas de famille sur laquelle elles pourraient compter. Leurs camarades se démènent de la même manière qu'elles pour survivre. Elles ne profitent aucunement des multiples bienfaits de nos sociétés modernes: l'accès aux soins médicaux, un logement décent, l'activité sportive, la musique, le cinéma ou le théâtre, ou encore les communications par Internet, en somme tout ce qui façonne nos sociétés contemporaines. Et ces personnes sont assurément «laissées pour compte» dans les réformes politiques visant le «bien commun», mais se traduisant dans les 
faits par le meilleur bénéfice possible pour le plus grand nombre de gens possible, et non par le meilleur bénéfice possible pour chaque individu. Voilà la différence entre une justice fondée sur une théorie qui prône une économie utilitariste et une justice fondée sur une théorie qui prône l'égalité des droits pour chaque individu.

La théorie de la reconnaissance, comme j'aimerais l'interpréter, rend chaque personne visible et audible de telle sorte qu'elle ne soit pas littéralement oubliée dans l'inévitable côté abstrait de toute théorie sur la justice. Une éthique basée sur une théorie politique critique que l'on associe avec le concept de reconnaissance n'est pas seulement une autre façon d'accepter la diversité des identités individuelles, ou d'accepter les politiques identitaires touchant les groupes sociaux. En fait, la reconnaissance ne peut être séparée d'une éthique du respect. Son objectif est manifestement le même que celui d'une éthique égalitaire, soit de donner à chaque personne la possibilité de vivre une vie agréable, de construire des sociétés tolérantes pouvant se côtoyer en paix, et d'établir des institutions politiques donnant à chaque personne la chance d'être entendue. Cependant, le côté critique de sa méthodologie transforme sa vision utopique - souvent idéalisée d'une société juste en analyse de l'injustice: au lieu de concevoir une societas perfecta, elle utilise un «négativisme méthodologique». Une éthique chrétienne utilisera donc une herméneutique classique, qui prête attention aux expériences négatives d'injustice, de honte et d'exclusion, et qui les prend au sérieux. Ainsi la considération de ces expériences deviendra un aspect essentiel de la théorie - ce qui est aussi de la théologie car, comme Metz l'estime, cette approche détermine comment parler de Dieu et à Dieu: «To speak of God means to express the suffering of others and to lament responsibility neglected and solidarity refused ».

Comme le dit Metz, ce sont les personnes souffrantes qui font autorité, et elles ne peuvent ni ne doivent être rejetées. Écouter leurs récits et leur porter une réelle attention nécessitent que l'on fasse disparaître l'anonymat des structures, et que l'on fasse disparaître les distances résultant de la mondialisation - cela implique donc de se rapprocher de ces personnes, et de les laisser s'approcher de nous; en d'autres mots, il nous faut traverser la rue pour les rencontrer, et leur tendre la main, semblablement à ce que fait si souvent Jésus dans les récits bibliques.

Conséquemment, la compassion pourrait très bien être la première attitude à adopter quand nous entendons un récit comme celui de Maria. Mais il faut aller au-delà de la compassion et transformer celle-ci en gestes de solidarité et de justice. À la manière de Nagel, il importe que nous recon- 
naissions que Maria a ses propres préoccupations, ses propres désirs et ses propres intérêts, comme chacun et chacune d'entre nous, ce qui signifie que nous la reconnaissions comme notre égale - ou plus exactement, ce qui importe avant tout est de reconnaître son humanité et sa capacité à faire ses propres choix moraux. Une solidarité avec Maria entraînerait de s'abstenir d'appeler trop rapidement un péché, qu'elle devrait immédiatement cesser de commettre, le commerce qu'elle fait de son corps. Une solidarité ne consiste pas à aborder Maria pour lui donner les quelques euros qu'elle gagnerait au cours d'une journée (bien que cela pourrait faire partie de notre geste de compassion). Ce serait plutôt de l'écouter avec attention, de comprendre les causes de sa condition et de faire pression pour que l'on modifie les réformes qui aident les établissements financiers et les instances qui y sont liées, mais en oubliant les personnes dont l'État a l'obligation de s'occuper. Cela veut aussi dire d'accompagner Maria dans quelques étapes afin qu'elle reprenne espoir. Tout cela fait partie des actions de solidarité que les communautés chrétiennes démontrent chaque jour, dans plusieurs régions du monde. Comme la célébration de la présence de Dieu dans le monde, cette pratique témoigne qu'il est possible, efficace et politiquement faisable de faire des choix moraux, fondés sur le principe égalitaire de justice et sur la réponse compatissante de solidarité avec les personnes souffrantes. En prenant encore une fois Metz comme modèle, je souligne ceci: une théologie chrétienne qui se préoccupe d'abord de la souffrance au lieu de la culpabilité peut mieux comprendre pourquoi il n'y a pas de justice sans solidarité - et vice-versa, pourquoi il ne peut y avoir de solidarité sans actions pour établir la justice.

Comme nous le savons tous, les personnes qui souffrent d'injustice et de manque de reconnaissance sont plus expertes que les spécialistes en éthique sur les questions qui les concernent. Mais lorsque leurs voix ne sont pas entendues, les théologiens, entre autres, peuvent - et parfois doivent - parler en leurs noms, et défendre leurs récits devant des personnes ayant d'autres intérêts. Pour conclure, la diversité doit avoir comme standard de permettre à chaque personne d'avoir les mêmes droits. Elle ne doit pas avoir le rôle d'un pavillon sous lequel naviguent l'inégalité et l'injustice au XXI ${ }^{\mathrm{e}}$ siècle. 


\section{Références}

HAKer, H. (1999), Moralische Identität. Literarische Lebensgeschichten als Medium ethischer Reflexion. Mit einer Interpretation der "Jahrestage" von Uwe Johnson, Tübingen, Francke.

Honneth, A. (1996), The Struggle for Recognition: The Moral Grammar of Social Conflicts. Studies in Contemporary German Social Thought, Cambridge, MIT Press.

(2011), Das Recht der Freiheit. Grundriss einer demokratischen Sittlichkeit, Frankfurt, Suhrkamp.

Metz, J. B. (1999), «Pluralism of Religious and Cultural Worlds: Notes toward a Theological and Political Program ", Cross Currents, 49/2, p. 227-236.

Ricoeur, P. (2007), Reflections on the Just, Chicago, University of Chicago Press.

Rawls, J. (1999), A Theory of Justice, Cambridge, Belknap Press of the Harvard University Press.

Press.

(2013), Political Liberalism, New York, Columbia Universty

\section{Résumé}

En commençant par déplorer l'absence de place pour la solidarité dans l'interprétation de la "justice de marché" au Xxie siècle, l'article propose une autre voie, soit la relation dialectique entre la justice et la solidarité. Alors que la justice met l'accent sur l'«égalité » pour tous, la solidarité s'attarde surtout sur la «différence» et la diversité. Le pont entre les deux concepts peut être trouvé dans les récentes réinterprétations de la théorie de la reconnaissance, qui touche les sphères privée, sociale et politique. L'article préconise toutefois une approche critique, qui prend le manque de reconnaissance, plutôt que la reconnaissance, comme point de départ. L'éthique en général et l'éthique chrétienne en particulier doivent prêter attention aux expériences et aux récits des personnes qui semblent invisibles et inaudibles dans les systèmes de justice fondés sur le mérite et les accomplissements, et ce, dans le but d'identifier les violations de droits aussi bien que les pratiques d'exclusion subies par ces personnes. En se préoccupant d'abord des expériences réelles d'injustice au lieu de la théorie normative de la justice, la relation dialectique entre la justice et la solidarité devient claire: la solidarité révèle non seulement le fossé entre la théorie et la pratique, ou l'inévitable côté aveugle de la justice, mais elle s'affiche aussi clairement en faveur des 
personnes souffrant d'injustice. La solidarité est orientée vers l'action. Elle fait pression sur les institutions pour qu'elles deviennent justes, tout en prônant des changements pour ceux et celles qui souffrent le plus d'injustice, et tout en accompagnant ces personnes. Par ailleurs, la solidarité critique mais aussi partisane nécessite la vision impartiale et égalitaire d'une justice en tant que critique d'une idéologie potentielle qui est liée à un concept identitaire et à une politique identitaire.

\section{Abstract}

Starting with the unfortunate lack of a place for solidarity in the $X X I^{\text {st }}$ century interpretation of "market justice", this paper spells out the alternative path, namely the dialectic of justice and solidarity. While justice emphasizes the "equality" of all, solidarity emphasizes "difference" and diversity. The bridge between the two concepts may be found in recent re-interpretations of recognition theory which embraces the personal, the social, and the political sphere. The paper insists, however, on a critical approach that takes misrecognition, rather than recognition, as its starting point. Ethics in general and Christian ethics in particular needs to attend to the experiences and narratives of those who are invisible and inaudible in approaches to justice based on merit and achievement, in order to identify violations of their rights as well as practices of stigmatization. Starting with experiences of injustice rather than with the normative theory of justice, the dialectic relation between justice and solidarity becomes clear: solidarity reveals not only the gap between theory and practice or the necessarily blind side of justice, but it also takes a clear stance in favour of those who suffer from injustice. Solidarity is action-oriented, seeking to force institutions to become just, while supporting of advocating change with and for those who suffer most from injustice. On the other hand, the critical but also partisan solidarity needs the impartial, equalitarian perspective of justice as critique of the potentially ideology that is linked to any identity concept and identity politics. 